\title{
A Method For Fine Resolution Frequency Estimation From Three DFT Samples
}

\author{
Çag̃atay Candan
}

\begin{abstract}
The parameter estimation of a complex exponential waveform observed under white noise is typically tackled in two stages. In the first stage, a coarse frequency estimate is found by the application of an N-point DFT to the input of length $N$. In the second stage, a fine search around the peak determined in the first stage is conducted. The method proposed in this paper presents a simpler alternative. The method suggests a nonlinear relation involving three DFT samples already calculated in the first stage to produce a real valued, fine resolution frequency estimate. The estimator approaches Jacobsen's estimator for large $N$ and presents a bias correction which is especially important for small and medium values of $N$.
\end{abstract}

Index Terms-Frequency estimation, fine doppler estimation, radar signal processing, DFT.

\section{INTRODUCTION}

$\mathbf{T}$ HE parameter estimation of a complex exponential observed under white noise is a fundamental signal processing problem which is central to many applications including spectrum estimation, array signal processing and radar signal processing. The computational requirements of the estimator are especially critical in radar signal processing applications where millions of hypothesis tests involving complex exponentials can be evaluated every second, [1].

It is well known that when the observation noise is white and Gaussian, the maximum likelihood frequency estimate of a single complex exponential waveform is the peak location of the Discrete-Time Fourier Transform (DTFT) of the received signal. Since DTFT computation over the continuum of $[0,2 \pi]$ is a formidable operation, the samples of the DTFT are calculated using the Discrete Fourier Transform (DFT). Typically an N-point DFT is calculated for the data length of $N$ samples leading to a resolution of $2 \pi / N$ on the frequency estimate.

In many applications, it is desirable to increase the resolution of the frequency estimate at the cost of some additional computation. As described in [2], a two-stage search can be implemented to improve the frequency estimate. First a coarse search with an N-point DFT is executed and then a fine search is implemented around the vicinity of the peak determined in the first

Manuscript received February 03, 2011; revised March 14, 2011; accepted March 24, 2011. Date of publication April 05, 2011; date of current version April 14, 2011. The associate editor coordinating the review of this manuscript and approving it for publication was Dr. Arumugam Nallanathan.

The author is with the Department of Electrical and Electronics Engineering, Middle East Technical University (METU), Ankara, Turkey. (e-mail: ccandan@metu.edu.tr).

Color versions of one or more of the figures in this paper are available online at http://ieeexplore.ieee.org.

Digital Object Identifier 10.1109/LSP.2011.2136378

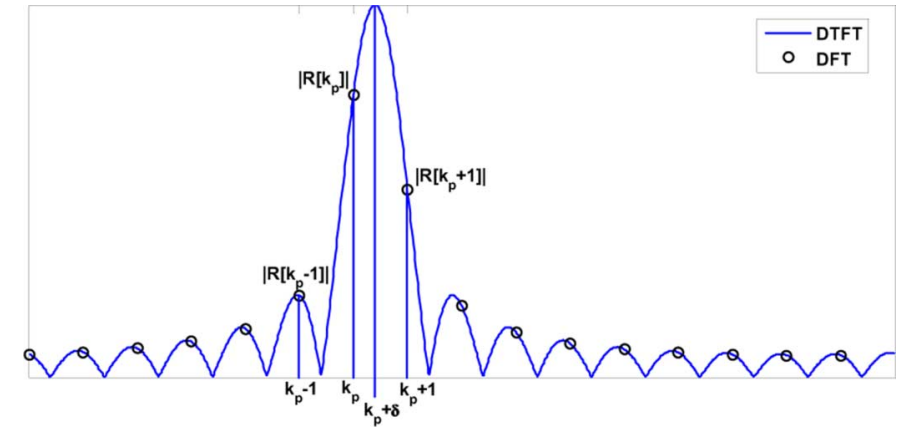

Fig. 1. Magnitude spectrum of the complex exponential waveform with frequency $\omega=2 \pi / N\left(k_{p}+\delta\right)$ radians per sample.

stage. It should be noted that the resolution of a two-stage search is limited to the spacing of the grid points used in fine search.

In [3]-[6] an alternative for the second stage is suggested. Instead of a grid search, the fine resolution estimate is produced through a function on DFT samples already calculated in the first stage. The methods suggested in [3]-[5] use three DFT samples, while the method of Provencher uses only two DFT samples, [6]. These methods require very few operations in comparison to the grid search and produce a real valued estimate for the frequency, instead of a discrete grid point.

In [7], Jacobsen has suggested a simple relation for DFT domain fine frequency estimation. The suggestion is based on empirical observations and presented without a proof. In this paper, we present a derivation for the Jacobsen formula and present a bias correction. The correction term is effective for high SNR values, but it comes at almost no additional computational cost and therefore can be used at any SNR level.

\section{PROBlem DESCRIPTION}

A single complex exponential waveform observed under white Gaussian noise can be modeled as follows:

$$
r[n]=A e^{j \omega n}+w[n]
$$

Here $A$ and $\omega$ are unknown parameters which are complex valued amplitude and real valued frequency with the unit of radians per sample, respectively.

Fig. 1 shows the magnitude spectrum of $r[n]$ for the noiseless case. The frequency of the complex exponential shown in Fig. 1 is $\omega=2 \pi / N\left(k_{p}+\delta\right)$. Our goal in this paper is to estimate $\delta$, where $|\delta|<1 / 2$, from three samples around the peak in the DFT spectrum.

In the first stage, an N-point DFT of $r[n]$ is calculated, $R[k]=$ $\sum_{n=0}^{N-1} r[n] e^{-j 2 \pi / N n k}$. Here $R[k]$ denotes the complex valued DFT output. The peak value in the DFT magnitude spectrum $\left(k_{p}\right.$ in Fig. 1) is expected to be around the true frequency $\omega$, if the 
input SNR $=|A|^{2} / \sigma_{w}^{2}$ is sufficiently large. Our interest in this paper is the estimation of the frequency in the high SNR region, which is a typical setting for a radar signal processing problem.

We can express the DFT bin where the peak occurs and its immediate left and right neighbors as follows:

$$
\begin{aligned}
R\left[k_{p}-1\right] & =A \sum_{n=0}^{N-1} e^{j \frac{2 \pi}{N}(\delta+1) n}+\tilde{w}[k-1] \\
& =A f(\delta+1)+\tilde{w}\left[k_{p}-1\right] \\
R\left[k_{p}\right] & =A \sum_{n=0}^{N-1} e^{j \frac{2 \pi}{N} \delta n}+\tilde{w}[k] \\
& =A f(\delta)+\tilde{w}\left[k_{p}\right] \\
R\left[k_{p}+1\right] & =A \sum_{n=0}^{N-1} e^{j \frac{2 \pi}{N}(\delta-1) n}+\tilde{w}[k+1] \\
& =A f(\delta-1)+\tilde{w}\left[k_{p}+1\right] .
\end{aligned}
$$

Here $\tilde{w}[k]$ is the DFT of $w[n]$ which is also white and jointly Gaussian distributed.

The function $f(\cdot)$ appearing on the right hand side of the equations given in (1) is defined as follows:

$$
f(\alpha)=\sum_{n=0}^{N-1} e^{j \frac{2 \pi}{N} \alpha n}
$$

Here $\alpha$ is a generic variable for the function $f(\cdot)$.

Our goal is to utilize three DFT samples around the peak to produce an estimate of $\delta$. Once $\delta$ is estimated, the final frequency estimate becomes $\hat{\omega}=2 \pi / N\left(k_{p}+\hat{\delta}\right)$ where $k_{p}$ is the bin number of the peak found in the first stage and $\hat{\delta}$ is the estimate produced by $R\left[k_{p}-1\right], R\left[k_{p}\right]$ and $R\left[k_{p}+1\right]$.

\section{PRoposed Estimator}

To determine $\delta$ from the set of nonlinear relations given in (1), we suggest to expand $f(\delta)$ in terms of its Taylor series around $\delta=0$ and solve for $\delta$ with the assumption of second and higher powers of $\delta$ being negligible in comparison to $\delta$.

We initiate the derivation with the Taylor series expansion of $g(z)=\sum_{n=0}^{N-1} e^{z n}:$

$$
\begin{aligned}
g(z) & =\sum_{n=0}^{N-1} e^{z n}=\sum_{n=0}^{N-1} \sum_{k=0}^{\infty} \frac{(z n)^{k}}{k !}=\sum_{k=0}^{\infty} \frac{z^{k}}{k !} \underbrace{\sum_{n=0}^{N-1} n^{k}}_{S_{k}(N-1)} \\
& =\sum_{k=0}^{\infty} \frac{z^{k}}{k !} S_{k}(N-1) .
\end{aligned}
$$

The function $S_{k}(N-1)$ denotes the summation of the $k$ th power of integers between 0 and $N-1$, that is $S_{k}(N-1)=0^{k}+$ $1^{k}+\cdots+(N-1)^{k}$. The first few terms of sequence $S_{k}(N)$ are given as follows:

$$
\begin{aligned}
S_{0}(N) & =N+1 \\
S_{1}(N) & =\frac{N(N+1)}{2} \\
S_{2}(N) & =\frac{N(N+1)(2 N+1)}{6} .
\end{aligned}
$$

The higher order terms of $S_{k}(N)$ can be written explicitly in terms of Bernoulli numbers, [8].

The Taylor series expansion of $f(\alpha)$ given in (2) can be immediately written by replacing $z$ with $j 2 \pi / N \alpha$ in $g(z)$ :

$$
\begin{aligned}
f(\alpha)=\sum_{k=0}^{\infty}(-1)^{k} \underbrace{}_{c_{2 k}} \underbrace{\frac{S_{2 k}(N-1)(2 \pi)^{2 k}}{(2 k) ! N^{2 k}}}_{c_{2 k+1}} \alpha^{2 k} \\
\quad+j \sum_{k=0}^{\infty}(-1)^{k} \underbrace{\underbrace{2 k+1} .}_{\underbrace{\frac{S_{2 k}(N-1)(2 \pi)^{2 k+1}}{(2 k+1) ! N^{2 k+1}}}_{2 k+1}}
\end{aligned}
$$

With the following definition of $c_{k}$

$$
c_{k}=\frac{S_{k}(N-1)(2 \pi)^{k}}{(k) ! N^{k}}
$$

the Taylor series can be written in a tidier form as follows:

$$
\begin{aligned}
f(\alpha)= & \sum_{k=0}^{\infty} c_{k}(j \alpha)^{k} \\
= & \left(c_{0}-c_{2} \alpha^{2}+c_{4} \alpha^{4}+\cdots\right) \\
& +j\left(c_{1} \alpha-c_{3} \alpha^{3}+c_{5} \alpha^{5}+\cdots\right) .
\end{aligned}
$$

To estimate $\delta$, we evaluate the first and second differences of $f(\delta)$. Using the elementary algebra, (8) can be written for the differences of $f(\delta+1)-f(\delta-1)$ and $f(\delta+1)-2 f(\delta)+f(\delta-1)$ Here the term h.o.t. denotes the higher order terms of $\delta$ :

$$
\begin{aligned}
f(\delta+1)-f(\delta-1) & \\
= & 2\left\{\left(-2 c_{2}+4 c_{4}-6 c_{6}+8 c_{8}+\cdots\right) \delta\right. \\
& \left.+j\left(c_{1}-c_{3}+c_{5}+\cdots\right)\right\}+ \text { h.o.t } \\
f(\delta+ & 1)-2 f(\delta)+f(\delta-1) \\
= & 2\left\{\left(-c_{2}+c_{4}-c_{6}+c_{8}+\cdots\right)\right. \\
& \left.+j\left(-3 c_{3}+5 c_{5}-7 c_{7} \ldots\right) \delta\right\}+ \text { h.o.t }
\end{aligned}
$$

Next, we evaluate the infinite summations involving odd and even indexed $c_{k}$ terms appearing on the right hand side of (8).

The infinite summations can be summed through the following relations:

$$
\begin{aligned}
& \sum_{k=0}^{\infty} c_{k} j^{k} \stackrel{(6)}{=} f(1) \stackrel{(2)}{=} \sum_{n=0}^{N-1} e^{j \frac{2 \pi}{N} n}=0 \\
& \sum_{k=1}^{\infty} k c_{k} j^{k} \stackrel{(6)}{=} f^{\prime}(1) \stackrel{(2)}{=} j \frac{2 \pi}{N} \sum_{n=0}^{N-1} n e^{j \frac{2 \pi}{N} n}=\pi \cot \left(\frac{\pi}{N}\right)-j \pi
\end{aligned}
$$

The two equations given in (9) can also be rewritten as shown in (10):

$$
\begin{aligned}
& \left(c_{0}-c_{2}+c_{4}-c_{6}+c_{8}+\ldots\right) \\
& \quad+j\left(c_{1}-c_{3}+c_{5}+\ldots\right)=0 \\
& \left(-2 c_{2}+4 c_{4}-6 c_{6}+8 c_{8}+\ldots\right) \\
& \quad+j\left(c_{1}-3 c_{3}+5 c_{5}+\ldots\right)=\pi \cot (\pi / N)-j \pi .
\end{aligned}
$$

From the definition of $c_{k}$, it can be easily seen that $c_{0}=N$, $c_{1}=\pi(N-1)$. Substituting $c_{0}$ and $c_{1}$ into the relations given 
TABLE I

Methods For Fine Resolution FREQUENCY Estimation IN DFT Domain

\begin{tabular}{|c|l|}
\hline Parabolic Interpolation [1] & $\widehat{\delta}=\left(\left|R_{k+1}\right|-\left|R_{k-1}\right|\right) /\left(4\left|R_{k}\right|-2\left|R_{k-1}\right|-2\left|R_{k+1}\right|\right)$ \\
\hline Quinn, [4] & $\alpha_{1}=\operatorname{Real}\left(R_{k-1} / R_{k}\right), \alpha_{2}=\operatorname{Real}\left(R_{k+1} / R_{k}\right)$ \\
& $\delta_{1}=\alpha_{1} /\left(1-\alpha_{1}\right), \delta_{2}=\alpha_{2} /\left(1-\alpha_{2}\right)$ \\
& if $\delta_{1}>0$ and $\delta_{2}>0, \widehat{\delta}=\delta_{2}$ \\
& else $\quad \widehat{\delta}=\delta_{1}$ \\
\hline MacLeod, [3] & $d=\operatorname{Real}\left(R_{k-1} R_{k}^{*}-R_{k+1} R_{k}^{*}\right) / \operatorname{Real}\left(2\left|R_{k}\right|^{2}+R_{k-1} R_{k}^{*}+R_{k+1} R_{k}^{*}\right)$ \\
& $\widehat{\delta}=\left(\sqrt{1+8 d^{2}}-1\right) /(4 d)$ \\
\hline Jacobsen, [7] & $\widehat{\delta}=\operatorname{Real}\left\{\left(R_{k-1}-R_{k+1}\right) /\left(2 R_{k}-R_{k-1}-R_{k+1}\right)\right\}$ \\
\hline Jacobsen with Bias Correction & $\widehat{\delta}=\frac{\tan (\pi / N)}{\pi / N} \operatorname{Real}\left\{\left(R_{k-1}-R_{k+1}\right) /\left(2 R_{k}-R_{k-1}-R_{k+1}\right)\right\}$ \\
\hline
\end{tabular}

in (10), we can explicitly evaluate the infinite summations in (8) as follows:

$$
\begin{aligned}
f(\delta+1)- & f(\delta-1) \\
& =2\{\pi \cot (\pi / N) \delta\}+\text { h.o.t } \\
f(\delta+1)- & 2 f(\delta)+f(\delta-1) \\
= & 2\{-N-j \pi N \delta\}+\text { h.o.t. }
\end{aligned}
$$

By ignoring the higher order terms, we get the following relation for the ratio of first two differences:

$$
\begin{aligned}
\frac{f(\delta+1)-f(\delta-1)}{f(\delta+1)-2 f(\delta)+f(\delta-1)}= & \frac{\pi \cot (\pi / N) \delta}{-N-j \pi N \delta} \\
= & \frac{-\pi N \cot (\pi / N)}{N^{2}+\pi^{2} N^{2} \delta^{2}} \delta \\
& +j \frac{\pi^{2} N \cot (\pi / N)}{N^{2}+\pi^{2} N^{2} \delta^{2}} \delta^{2} .
\end{aligned}
$$

Invoking the smallness of $\delta^{2}$ with respect to $\delta$ one more time, we can simplify the last relation as follows:

$$
\text { Real }\left\{\frac{f(\delta+1)-f(\delta-1)}{f(\delta+1)-2 f(\delta)+f(\delta-1)}\right\}=\frac{-\pi \cot (\pi / N)}{N} \delta
$$

In the high SNR regime, that is when $|A|^{2} \gg \sigma_{w}^{2}$, DFT samples around the peak value, that is $R\left[k_{p}-1\right], R\left[k_{p}\right]$, and $R\left[k_{p}+\right.$ $1]$ in (1), can be taken as $A f(\delta+1), A f(\delta)$ and $A f(\delta-1)$ respectively. Then an estimate for $\delta$ can be produced via the substitution of $f(\delta) \rightarrow R\left[k_{p}\right], f(\delta-1) \rightarrow R\left[k_{p}+1\right]$ and $f(\delta+1) \rightarrow R\left[k_{p}-1\right]$ into (12):

$$
\hat{\delta}=\frac{\tan (\pi / N)}{\pi / N} \operatorname{Real}\left\{\frac{R\left[k_{p}-1\right]-R\left[k_{p}+1\right]}{2 R\left[k_{p}\right]-R\left[k_{p}-1\right]-R\left[k_{p}+1\right]}\right\} .
$$

This concludes the derivation of the proposed estimator. It should be noted that the estimator proposed approaches the estimator of Jacobsen, given in Table I, as $N \rightarrow \infty$.

\section{NUMERICAL COMPARISONS}

This section presents a numerical comparison of the proposed estimator with the other estimators given in Table I. We would

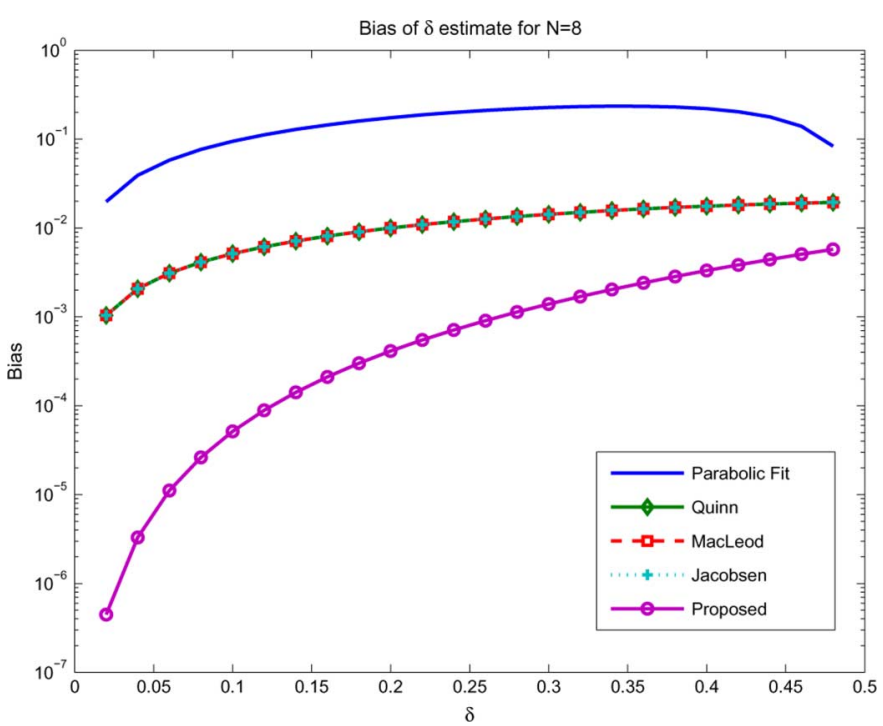

Fig. 2. Bias of different estimator in the absence of noise.

like to remind that in the presented figures, the bias and root mean square error (RMSE) values describe the error on $\hat{\delta}$. This error can be interpreted as the error normalized to the DFT bin size. With this normalization, an error of 0.1 units on $\hat{\delta}$ is equivalent to the frequency error of $2 \pi / N 0.1$ radians per sample.

Fig. 2 compares the bias of the proposed and other estimators in the absence of noise for $N=8$. Frequency estimation is a nonlinear estimation problem, therefore it is not surprising that all estimators, including the proposed one, are biased. As expected, the bias gets smaller as $N \rightarrow \infty$ or as SNR increases. Fig. 2 shows that the least biased estimator is the proposed one. (This is in general true for any $N$.) The poorest bias belongs to the parabolic fit estimator which is a fact known in the literature, [1]. The other estimators by Quinn, Jacobsen, and Macleod have the exact same bias value in the absence of noise.

Fig. 3 examines the bias in the presence of noise. For this figure, the parameter $\delta$ is fixed to a specific value, which is $\delta=$ 0.25 , and SNR is varied. As can be observed from this figure, the estimators approach the bias value of the noiseless case, which is presented in Fig. 2, as SNR increases.

Fig. 4 shows the RMSE of the estimators and the Cramer-Rao lower bound. We note that the Cramer-Rao bound is not typically applicable for the biased estimators, but it is still useful if the bias value is much smaller than the error variance. As can 


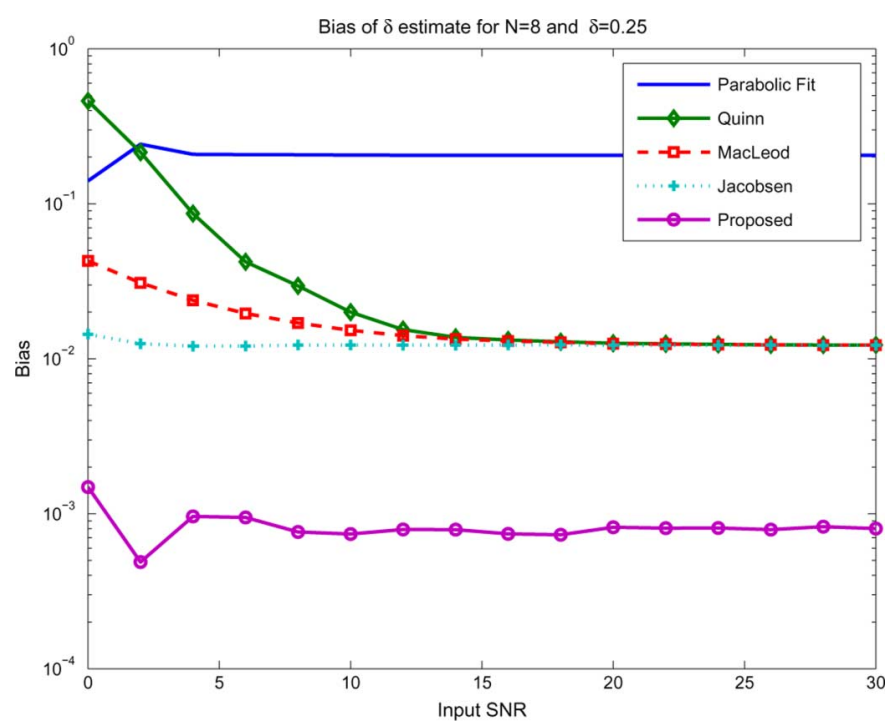

Fig. 3. Variation of bias with respect to SNR for $\delta=0.25$ and $N=8$.

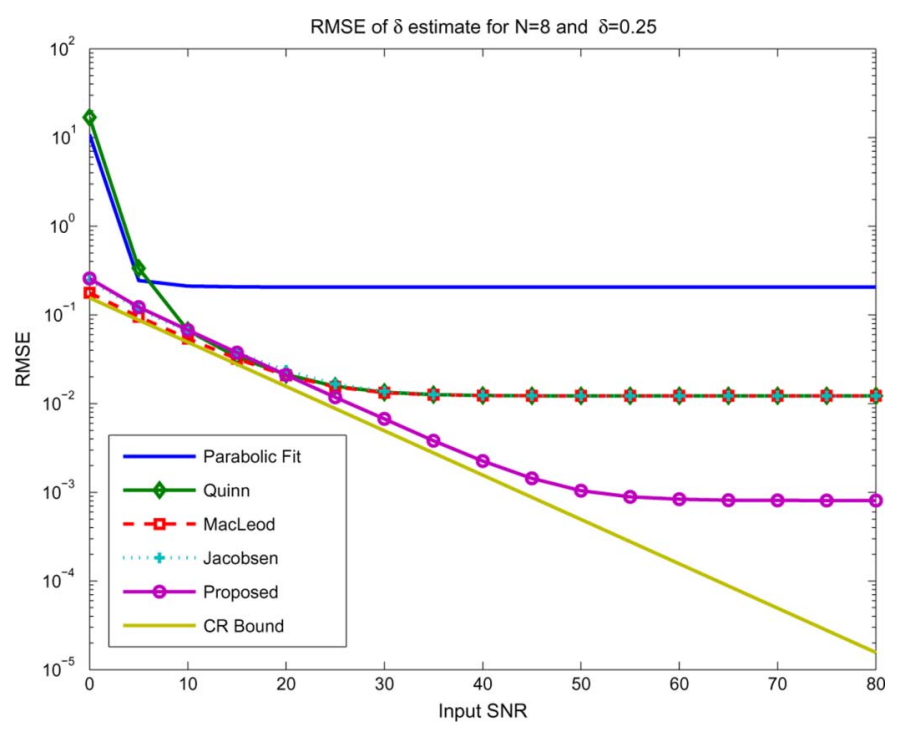

Fig. 4. Variation of RMSE with respect to SNR for $\delta=0.25$ and $N=8$.

be noted from this figure, the RMSE values of all estimators including the proposed one are dominated by the estimator bias at sufficiently high SNR values.

As a final comparison, we present a second RMSE comparison figure for a larger number of observation samples. Fig. 5 shows the RMSE for $N=32$. It can be noted from this figure that the RMSE floor occurs at a higher SNR value for $N=32$ than $N=8$. Other than this change, $N=32$ case follows $N=8$ case quite closely.

It should be noted that when $N$ is large, Jacobsen's formula and its bias corrected version has the same performance for a large SNR range which can encompass the SNR range of many practical applications. We believe that the correction is most useful for the applications with small/medium values for $N$ at high SNR such as the Doppler frequency estimation problem in radar signal processing.

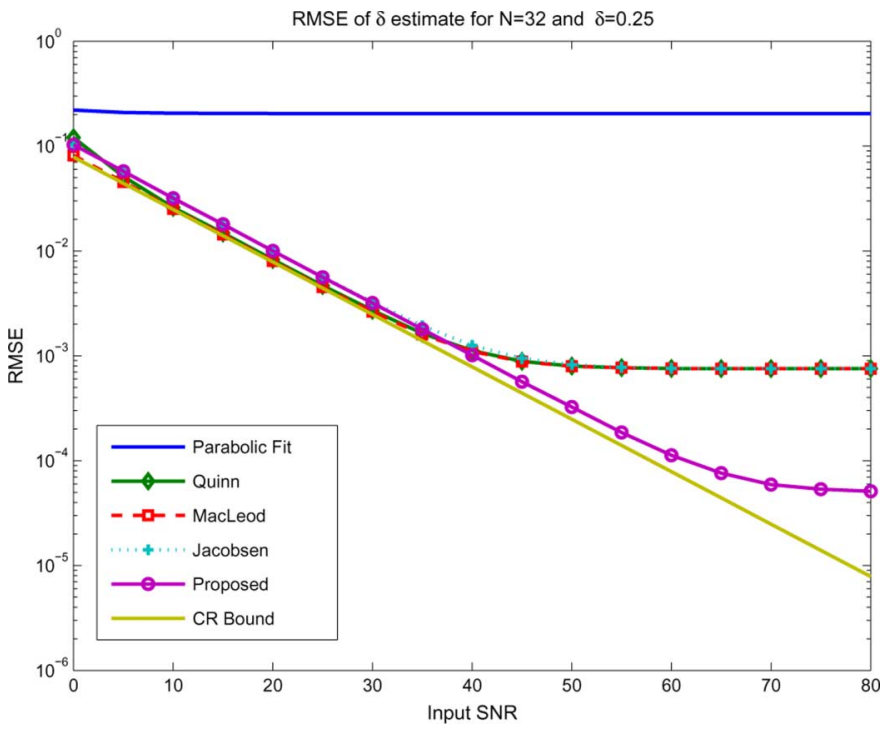

Fig. 5. Variation of RMSE with respect to SNR for $\delta=0.25$ and $N=32$.

\section{CONCLUSION}

We present a justification for the good performance of Jacobsen's estimator and suggest a bias correction further improving its performance. Jacobsen's estimator has been suggested through empirical observations in [7] and this work presents a justification for its good performance.

The proposed estimator requires very few operations per output sample and closely follows the Cramer-Rao bound in high SNR region. These features make the estimator especially valuable for radar signal processing applications where efficient and accurate computation of unknown target Doppler frequency is needed.

The present work utilizes the data processed by a rectangular window. A potential future work is the derivation similar fine resolution frequency estimation formulas for different windows such as Hamming, Hanning windows.

\section{ACKNOWLEDGMENT}

The author acknowledges comments and suggestions of $\mathrm{E}$. Jacobsen on the present paper.

\section{REFERENCES}

[1] M. A. Richards, Fundamentals of Radar Signal Processing. New York: McGraw-Hill, 2005.

[2] H. L. V. Trees, Detection, Estimation and Modulation Theory, Part 1. New York: Wiley, 1971.

[3] M. D. Macleod, "Fast nearly ML estimation of the parameters of real or complex single tones or resolved multiple tones," IEEE Trans. Signal Process., vol. 46, no. 1, pp. 141-148, Jan. 1998.

[4] B. G. Quinn, "Estimating frequency by interpolation using Fourier coefficients," IEEE Trans. Signal Process., vol. 42, no. 5, pp. 1264-1268, May 1994.

[5] B. G. Quinn, "Estimation of frequency, amplitude, and phase from the DFT of a time series," IEEE Trans. Signal Process., vol. 45, no. 3, pp. 814-817, Mar. 1997.

[6] S. Provencher, "Estimation of complex single-tone parameters in the DFT domain," IEEE Trans. Signal Processing, vol. 58, no. 7, pp. 3879-3883, 2010.

[7] E. Jacobsen and P. Kootsookos, "Fast, accurate frequency estimators," IEEE Signal Process. Mag., vol. 24, pp. 123-125, May 2007.

[8] D. Knuth, "Johann Faulhaber and sums of powers," Math. Comput., vol. 61, no. 203, pp. 277-294, 1993. 